Author affiliations and support information (if applicable) appear at the end of this article.

Published at jco.org on March 14, 2017 Clinical trial information: NCT00433433 Corresponding author: John Raemaekers, MD, PhD, Department of Hematology, Radboud University Medical Center, PO Box 9101, 6500HB Nijmegen, the Netherlands; e-mail: johnmienraemaekers@ gmail.com

๑) 2017 by American Society of Clinical Oncology

0732-183X/17/3516w-1786w/\$20.00

\title{
Early Positron Emission Tomography Response-Adapted Treatment in Stage I and II Hodgkin Lymphoma: Final Results of the Randomized EORTC/LYSA/FIL H10 Trial
}

Marc P.E. André, Théodore Girinsky, Massimo Federico, Oumédaly Reman, Catherine Fortpied, Manuel Gotti, Olivier Casasnovas, Pauline Brice, Richard van der Maazen, Alessandro Re, Véronique Edeline, Christophe Fermé, Gustaaf van Imhoff, Francesco Merli, Réda Bouabdallah, Catherine Sebban, Lena Specht, Aspasia Stamatoullas, Richard Delarue, Valeria Fiaccadori, Monica Bellei, Tiana Raveloarivahy, Annibale Versari, Martin Hutchings, Michel Meignan, and John Raemaekers

\section{$\begin{array}{lllllllll}\text { A } & \text { B } & S & \mathbf{T} & \mathbf{R} & \mathbf{A} & \mathbf{C} & \mathbf{T}\end{array}$}

\section{Purpose}

Patients who receive combined modality treatment for stage I and II Hodgkin lymphoma (HL) have an excellent outcome. Early response evaluation with positron emission tomography (PET) scan may improve selection of patients who need reduced or more intensive treatments.

\section{Methods}

We performed a randomized trial to evaluate treatment adaptation on the basis of early PET (ePET) after two cycles of doxorubicin, bleomycin, vinblastine, and dacarbazine (ABVD) in previously untreated-according to European Organisation for Research and Treatment of Cancer criteria favorable (F) and unfavorable (U)—stage I and II HL. The standard arm consisted of ABVD followed by involved-node radiotherapy (INRT), regardless of ePET result. In the experimental arm, ePETnegative patients received ABVD only (noninferiority design), whereas ePET-positive patients switched to two cycles of bleomycin, etoposide, doxorubicin, cyclophosphamide, vincristine, procarbazine, and prednisone (BEACOPPesc) and INRT (superiority design). Primary end point was progression-free survival (PFS).

\section{Results}

Of 1,950 randomly assigned patients, 1,925 received an ePET-361 patients (18.8\%) were positive. In ePET-positive patients, 5-year PFS improved from 77.4\% for standard ABVD + INRT to 90.6\% for intensification to BEACOPPesc + INRT (hazard ratio $[\mathrm{HR}], 0.42 ; 95 \% \mathrm{Cl}, 0.23$ to $0.74 ; P=.002$ ). In ePET-negative patients, 5-year PFS rates in the F group were $99.0 \%$ versus $87.1 \%(\mathrm{HR}, 15.8 ; 95 \%$ $\mathrm{Cl}, 3.8$ to 66.1$)$ in favor of $A B V D+I N R T$; the $U$ group, $92.1 \%$ versus $89.6 \%(\mathrm{HR}, 1.45 ; 95 \% \mathrm{Cl}, 0.8$ to 2.5) in favor of ABVD + INRT. For both $F$ and $U$ groups, noninferiority of ABVD only compared with combined modality treatment could not be demonstrated.

\section{Conclusion}

In stage I and II HL, PET response after two cycles of ABVD allows for early treatment adaptation. When ePET is positive after two cycles of ABVD, switching to BEACOPPesc + INRT significantly improved 5-year PFS. In ePET-negative patients, noninferiority of ABVD only could not be demonstrated: risk of relapse is increased when INRT is omitted, especially in patients in the F group.

\section{J Clin Oncol 35:1786-1794. (c) 2017 by American Society of Clinical Oncology}

\section{INTRODUCTION}

Treatment of early-stage Hodgkin lymphoma (HL) is extremely successful, with a more than $90 \%$ cure rate. The backbone of these results is a combination of chemotherapy and radiotherapy (RT $)^{1,2}$; however, late toxicities - mainly second malignancies ${ }^{3,4}$ and cardiovascular events_-are of major concern. ${ }^{5,6}$
Extended RT fields and alkylating agents are held responsible, and avoidance of RT has been tested in several randomized trials but is associated with an increased risk of early relapse. ${ }^{7}$ Conversely, a small, clinically relevant subgroup of patients experience failure with standard combined modality treatment (CMT) treatment. A sensitive tool to identify early those patients who have a high cure rate with current standards and 68.6394

DOI: https://doi.org/10.1200/JCO. 2016.68.6394

DOI: https://doi.org/10.1200/JCO.2016 
those who should switch to other treatment is a clear unmet need.

A positron emission tomography (PET) scan performed early during treatment, after two cycles of chemotherapy, predicts outcome when the preplanned treatment is continued: patients with a negative early PET (ePET) scan have the greatest chance of achieving cure ( $>90 \%$ ), whereas outcomes for those with a positive ePET scan is significantly worse. ${ }^{8-10}$ It is tempting to consider the ePET scan as the missing tool in selecting patients who can be treated less intensively, without RT, and those who need early intensified therapy; therefore, we designed a randomized trial in which patients with stage I and II HL were randomly assigned to either a standard CMT program, regardless of the result of the ePET, or to an experimental arm in which patients with a negative ePET scan were treated with chemotherapy alone and those with a positive ePET scan changed from conventional chemotherapy to an intensified schedule. This H10 Intergroup trial (European Organisation of Research and Treatment of Cancer [EORTC], Lymphoma Study Association, and Fondazione Italiana Linfomi) is the first and only trial, to our knowledge, to incorporate a randomized ePET response-adapted treatment strategy for both the ePET-negative and the ePET-positive patients with stage I and II HL. Results of the preplanned interim analysis of the ePETnegative groups of patients have been published. ${ }^{11}$ Here, we report the final analysis of all patients.

\section{METHODS}

\section{Eligibility}

Previously untreated patients, age 15 to 70 years, with classic supradiaphragmatic stage I and II HL were eligible. Both favorable (F) and unfavorable $(\mathrm{U})$ patients, according to EORTC criteria, ${ }^{1}$ were included (U: at least one of the following criteria: age $\geq 50$ years or more than three nodal areas or mediastinal-thoracic ratio $\geq 0.35$ or no $\mathrm{B}$ symptoms and erythrocyte sedimentation rate $[\mathrm{ESR}] \geq 50$ or $\mathrm{B}$ symptoms and $\mathrm{ESR} \geq 30$; F: all others). Staging was based on conventional computed tomography scanning $^{12}$; a baseline PET was recommended. The study was approved by the scientific and ethical committees and all patients gave written informed consent (full protocol in the Appendix SA1, online only).

\section{Study Design}

In this multicenter, phase III trial, patients were randomly assigned upfront in a 1:1 ratio to either standard CMT or experimental ePET response-adapted treatment. A minimization technique was used for treatment allocation in the $\mathrm{F}$ and $\mathrm{U}$ groups separately, stratifying by institution, Ann Arbor stage (I $v$ II), and availability of baseline PET. All patients received two cycles of doxorubicin, bleomycin, vinblastine, and dacarbazine (ABVD), after which an ePET was performed.

The primary objective of the study was to evaluate whether involvednode RT (INRT) could be omitted without loss of efficacy in ePET-negative patients after two cycles of ABVD. In the standard arm, ePET-negative patients received one (for $\mathrm{F}$ ) or two (for $\mathrm{U}$ ) additional ABVD cycles, followed by INRT. In the experimental arm, ePET-negative patients received two (for F) or four (for $\mathrm{U}$ ) additional ABVD cycles but no radiotherapy (Fig 1). ${ }^{11}$ This primary objective was evaluated in $\mathrm{F}$ and $\mathrm{U}$ patients separately.

The secondary objective was to evaluate whether intensification with escalated doses of bleomycin, etoposide, doxorubicin, cyclophosphamide, vincristine, procarbazine and prednisone (BEACOPPesc) after two cycles of ABVD would improve outcome in ePET-positive patients. In the

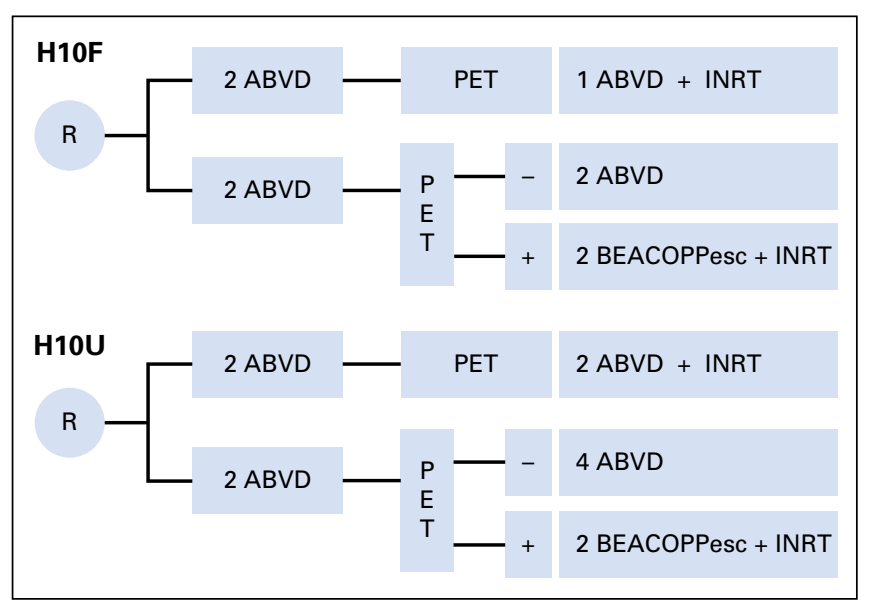

Fig 1. Study design. ABVD, doxorubicin, bleomycin, vinblastine, and dacarbazine; BEACOPPesc, bleomycin, etoposide, doxorubicin, cyclophosphamide, vincristine, procarbazine and prednisone; F, favorable; INRT, involved-node radiotherapy; PET, positron emission tomography; $U$, unfavorable.

standard arm, ePET-positive patients received one (for F) or two (for U) additional ABVD cycles, followed by INRT, whereas in the experimental arm, ePET-positive patients (both F and $\mathrm{U}$ ) switched to two cycles of BEACOPPesc followed by INRT (Fig 1). For this objective, ePET-positive F and $U$ patients were pooled because of their presumed common poor prognosis.

\section{Treatment}

ABVD and BEACOPPesc were administered as described previously. ${ }^{13}$ RT was delivered according to the EORTC INRT concept: only initially involved nodes were irradiated instead of whole areas. ${ }^{14,15}$ Training sessions were organized before the start of the trial in all participating countries, and a nonmandatory prospective quality assurance program was performed in France.

\section{PET Scanning}

ePET was scheduled between days 22 and 25 of the second cycle of ABVD. Images were scored according to International Harmonization Project criteria. ${ }^{16}$ According to these criteria, mediastinal blood pool activity is recommended as reference background activity to define PET positivity of a residual mass $\geq 2 \mathrm{~cm}$ in greatest transverse diameter, regardless of its location. A smaller residual mass or a normal-sized lymph node is considered positive if its activity is above that of the surrounding background. A prospective central review of ePET was planned. For the Lymphoma Study Association, central review was performed online from the start of the trial in 2006. ${ }^{17}$ For EORTC and Fondazione Italiana Linfomi, central review started 2 years later as a result of information technology system incompatibility problems. In the absence of a timely centralized reading, local ePET reports decided further treatment in the experimental arm.

\section{Statistical Design}

Primary end point was progression-free survival (PFS), definedfrom the date of random assignement to date of progression-as experiencing relapse after previous complete remission or progression after reaching partial remission ( $50 \%$ decrease and resolution of B symptoms and no new lesions); progressive disease (50\% increase from nadir of any previous partial remission lesions or appearance of new lesions) on computed tomography scan measurements during protocol treatment; or death from any cause, whichever occurred first. This objective was assessed via noninferiority test using a log-rank test stratified by stage (I $v$ II) and availability of baseline PET, with a one-sided significance level of .025. 
Assuming a 5-year PFS in the ePET-negative standard arm of $95 \%$ for F group and $90 \%$ for U group, as well as allowing for a decrease of $10 \%$, the resulting noninferiority margins for the hazard ratios (HRs) were 3.2 and 2.1 for $\mathrm{F}$ and $\mathrm{U}$ groups, respectively. As a result of the good prognosis of these patients, the absolute difference of $10 \%$ translates into noninferiority margins that are markedly higher than the noninferiority margins for HRs. Noninferiority is concluded if the upper bound of the 95\% CI for the estimated HR does not exceed the noninferiority margin. A total of 26 and 63 events (progressions or deaths) for F and U groups, respectively, were required for $80 \%$ power. The original protocol estimated that a total of 248 ePET-positive patients and a total of 77 events would be obtained simultaneously. This would give $77 \%$ power to detect a $20 \%$ improvement in 5 -year PFS (from $50 \%$ to $70 \%$ ) using a two-sided log-rank test $(\alpha=.05$ ).

The interim futility analysis of ePET-negative patients was previously reported and a safety amendment to close the ABVD only arms was issued in August 2010. ${ }^{11}$ At the same time, an interim efficacy analysis was performed on ePET-positive patients and the target accrual for ePETpositive patients was increased to 355 to reach the expected number of events in a reasonable timeframe. The study was closed without any interruption in recruitment in June 2011. In 2013, the independent data monitoring committee recommended that the final analysis be performed at least 3 years after the last patient was enrolled.

\section{Statistical Analysis}

Primary efficacy analyses were conducted on the intention-to-treat (ITT) principle in all patients with ePET result after the first two cycles of ABVD. Preplanned sensitivity analyses, including a per-protocol analysis, were also performed. The present noninferiority analysis on ePET-negative patients includes 900 patients who were included in the interim analysis ${ }^{11}$ as well as additional patients who were randomly assigned between the interim analysis and the safety amendment (overruns). ePET-negative patients included after the safety amendment and all who were treated with
CMT are not included in this final analysis. A Cox proportional hazards regression model was used to estimate treatment effect HRs and their 95\% CIs. Survival curves were estimated on the basis of the Kaplan-Meier method and 5-year survival estimates were provided, together with their 95\% CI. In ePET-positive patients, a two-sided superiority test for PFS was used with a significance level of .037 to account for the type I error spent at the interim analysis for early superiority.

\section{RESULTS}

\section{Patients}

From November 2006 to June 2011, 1,950 patients were enrolled (Fig 2). Clinical characteristics of 361 ePET-positive patients, 1,059 ePET-negative patients who were treated per the initial protocol, and 505 ePET-negative patients who were enrolled after the safety amendment are listed in Table 1. Twenty-five patients who were included did not receive ePET after two cycles of ABVD. Baseline recommended PET was performed in $96 \%$ of patients. Seventy-five percent of ePET scans were centrally reviewed, with $93 \%$ concordance with local assessment (Cohen's $\kappa=0.78 ; 95 \% \mathrm{CI}, 0.74$ to 0.82 ). ePET positivity was reported in $18.8 \%$ of patients: $13.0 \%$ and $22.4 \%$ in $\mathrm{F}$ and $\mathrm{U}$ groups, respectively.

\section{Outcome of ePET-Positive Patients}

In the overall ePET-positive group $(\mathrm{n}=361)$, after a median follow-up of 4.5 years, a total of 57 events for PFS occurred: $41-36$ relapses and 5 deaths not related to HL-in the ABVD + INRT arm

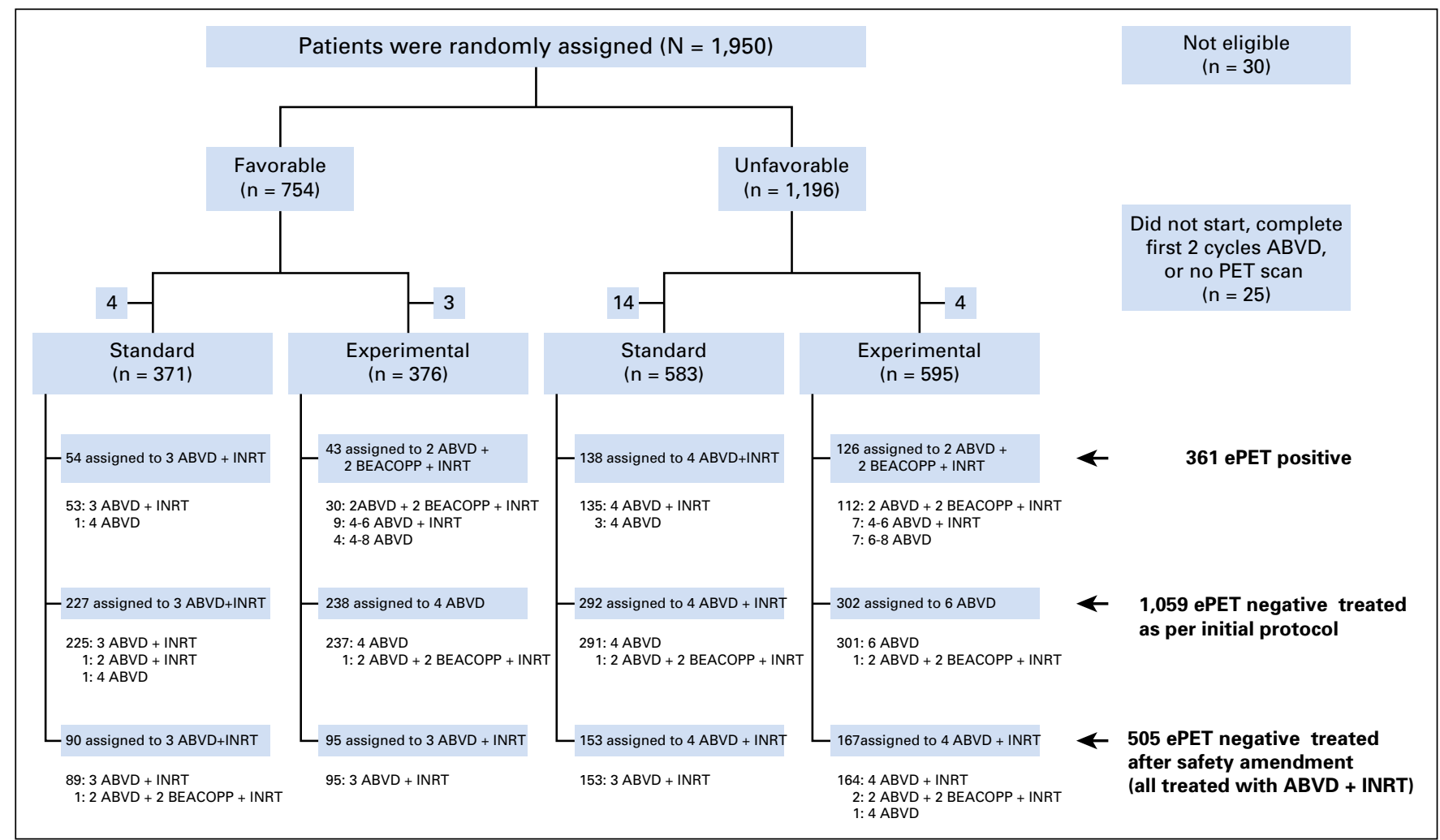

Fig 2. CONSORT diagram. ABVD, doxorubicin, bleomycin, vinblastine, and dacarbazine; BEACOPPesc, bleomycin, etoposide, doxorubicin, cyclophosphamide, vincristine, procarbazine, and prednisone; INRT, involved-node radiotherapy; PET, positron emission tomography. 


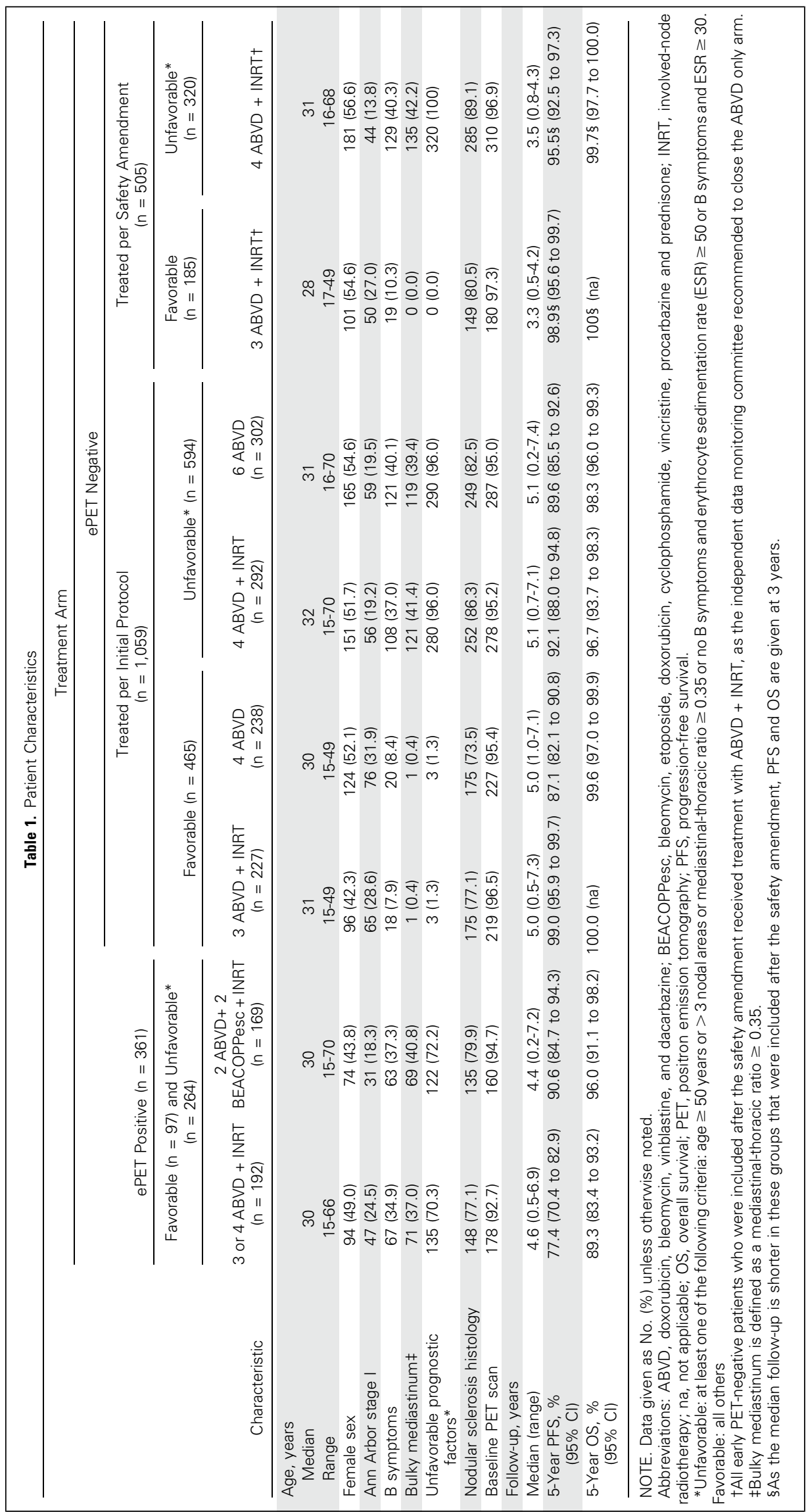


and $16-13$ relapses and 3 deaths not related to $\mathrm{HL}$-in the BEACOPPesc + INRT arm. ITT 5-year PFS rates were 77.4\% (95\% CI, 70.4 to 82.9$)$ and $90.6 \%(95 \% \mathrm{CI}, 84.7$ to 94.3$)$ in the ABVD + INRT and BEACOPPesc + INRT arms, respectively, with HR, 0.42 (95\%CI, 0.23 to $0.74 ; P=.002$ ) in favor of BEACOPPesc + INRT (Fig 3A).

In the ABVD + INRT arm, 23 patients experienced relapse exclusively in previously involved nodes, eight in previously uninvolved nodes, and five in both, whereas in the BEACOPPesc + INRT arm, seven patients experienced relapse exclusively in previously involved nodes, four in previously uninvolved nodes, and two in both.

The 5-year overall survival (OS) rates were $89.3 \%$ versus 96.0\% for ABVD + INRT and BEACOPPesc + INRT, respectively, with $\mathrm{HR}, 0.45$ (95\% CI, 0.19 to $1.07 ; P=.062$; Fig $3 \mathrm{~B}$ ).

Preplanned sensitivity analyses, including a per-protocol analysis, were performed and led to similar conclusions (Appendix Table A1, online only).

\section{Outcome of ePET-Negative Patients}

In the F group ( $\mathrm{n}=465)$, after a median follow-up of 5.0 years, a total of 33 events for PFS occurred: two patients experienced relapse in the ABVD + INRT arm versus 30 patients who experienced relapse and one patient who died from a cause not related to $\mathrm{HL}$ in the ABVD only arm. ITT 5-year PFS rates were $99.0 \%$ (95\% CI, 95.9 to 99.7$)$ and $87.1 \%(95 \% \mathrm{CI}, 82.1$ to 90.8$)$ in the ABVD + INRT and ABVD only arms, respectively, with HR, 15.8 (95\% CI, 3.8 to 66.1 ) in favor of ABVD + INRT (Fig 4A). Noninferiority could not be demonstrated as the upper bound of the $95 \%$ CI for the estimated HR (66.07) exceeded the prespecified noninferiority margin (3.2).

In the ABVD + INRT arm, no patient experienced relapse exclusively in previously involved nodes, one in previously uninvolved nodes, and one in both, whereas in the ABVD only arm, 22 patients experienced relapse exclusively in previously involved nodes, five in previously uninvolved nodes, and three in both.
Table 2 lists the causes of death. The 5-year OS rates were $100.0 \%$ versus $99.6 \%$ for ABVD + INRT and ABVD only arms, respectively (Appendix Fig A1A, online only).

In the U group $(\mathrm{n}=594)$, after a median follow-up of 5.1 years, a total of 54 events for PFS occurred: 16 patients experienced relapse and six died from causes not related to HL in the ABVD + INRT arm, whereas 30 patients experienced relapse and two died from causes not related to HL in the ABVD only arm. ITT 5-year PFS rates were 92.1\% (95\% CI, 88.0 to 94.8$)$ and $89.6 \%$ (95\% CI, 85.5 to 92.6) in the ABVD + INRT and ABVD only arms, respectively, with $\mathrm{HR}, 1.45$ (95\% CI, 0.8 to 2.5 ) in favor of ABVD + INRT (Fig 4B). Noninferiority could not be demonstrated as the upper bound of the 95\% CI for the estimated HR (2.50) exceeded the prespecified noninferiority margin (2.10).

In the ABVD + INRT arm, five patients experienced relapse exclusively in previously involved nodes, four in previously uninvolved nodes, and six in both, with one unknown, whereas in the ABVD only arm, 20 patients experienced relapse exclusively in previously involved nodes, four in previously uninvolved nodes, and six in both.

Causes of death are listed in Table 2. The 5-year OS rates were 96.7\% versus $98.3 \%$ for ABVD + INRT and ABVD only arms, respectively (Appendix Fig A1B). Preplanned sensitivity analyses, including a per-protocol analysis, were performed and led to similar conclusions (Appendix Table A1).

The outcomes of the 505 ePET-negative patients who were included after the safety amendment and all treated with CMT did not differ from those of the standard CMT in the randomized setting (Table 2).

\section{Treatment Compliance and Toxicity}

No unexpected toxicities were observed in ePET-negative patients in either arm.

In ePET-positive patients, overall, $8.6 \%$ did not start the allocated chemotherapy after ePET: four patients in the ABVD +
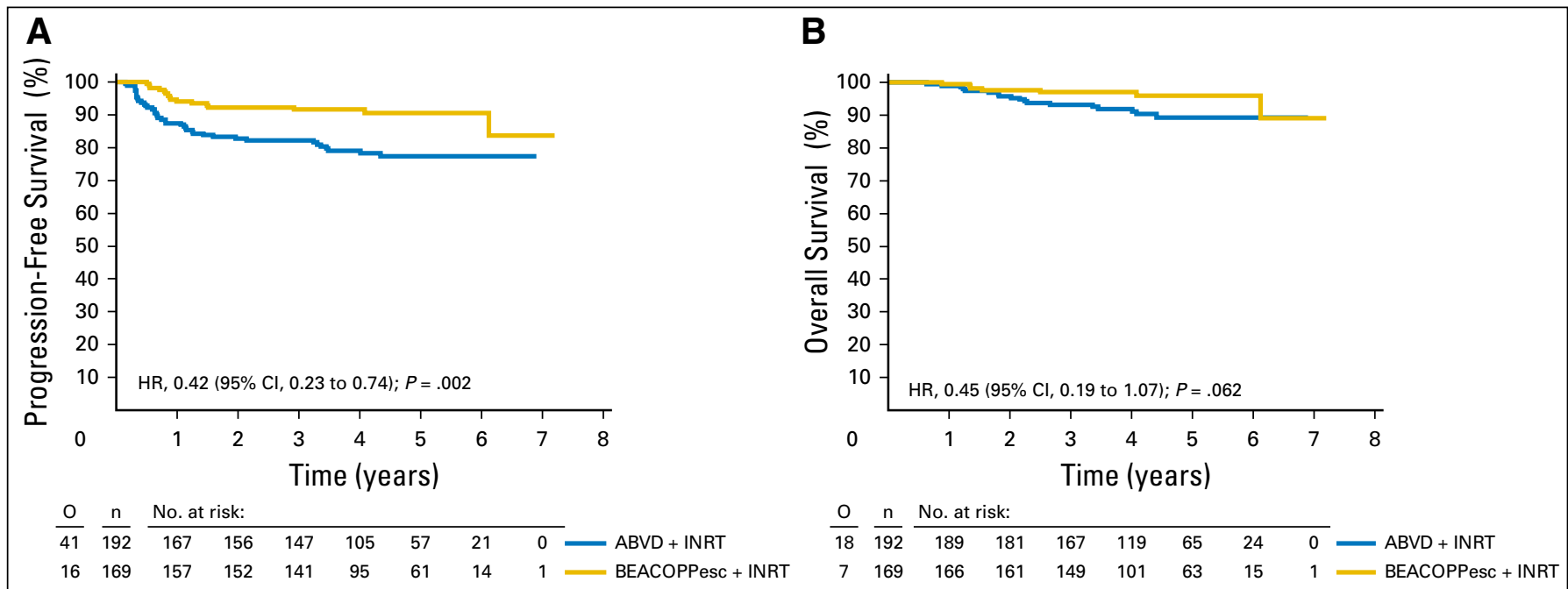

Fig 3. Progression-free and overall survival of early positron emission tomography (PET)-positive patients. Shown are the rates of ( $A$ ) progression-free and (B) overall

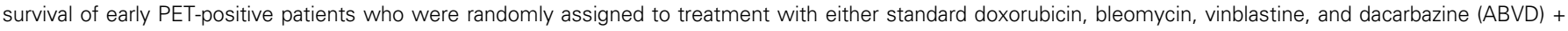

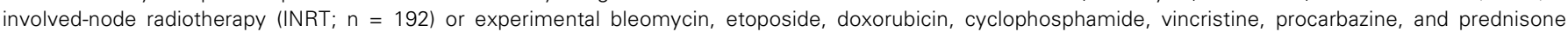
(BEACOPPesc) + INRT ( $\mathrm{n}=169)$. HR, hazard ratio, O observed; $\mathrm{n}$, number of patients. 


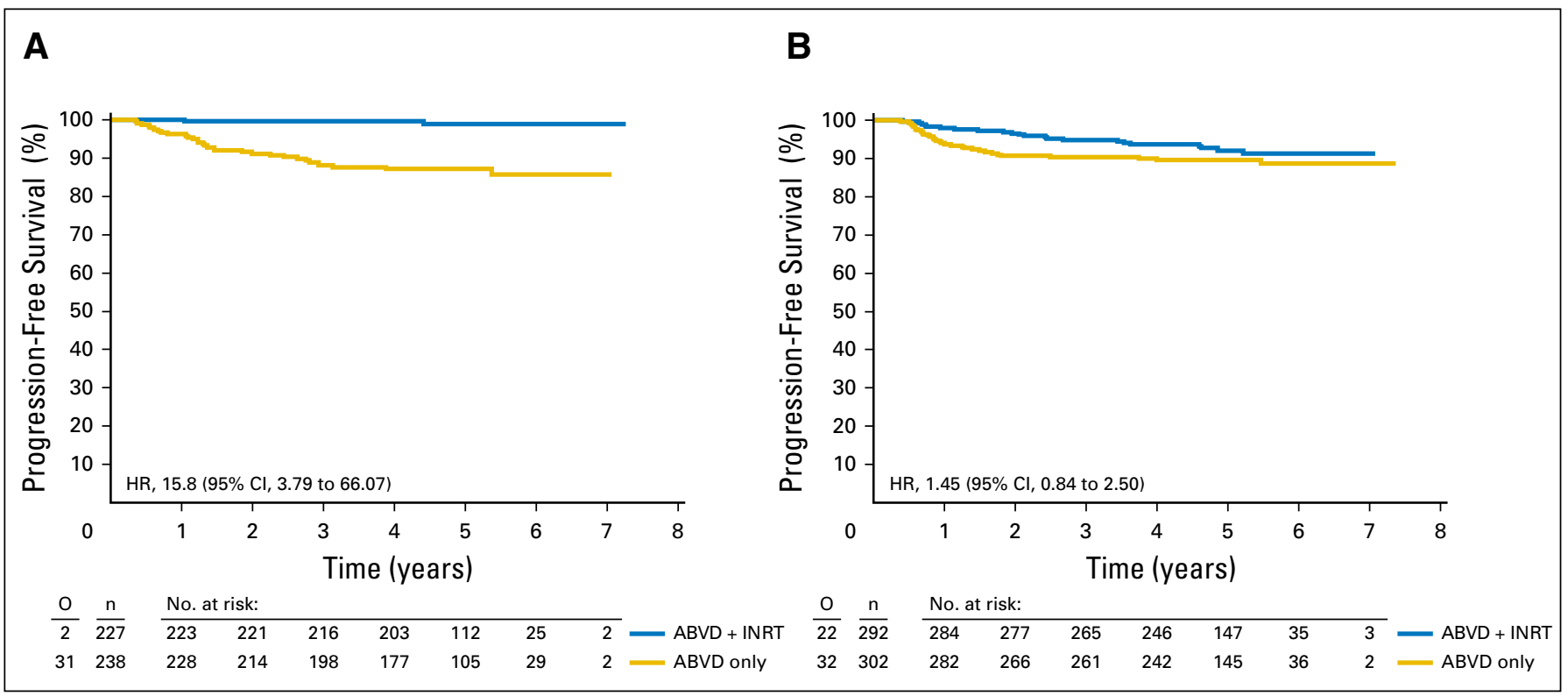

Fig 4. Progression-free survival of 1,059 early positron emission tomography-negative patients who were treated per the initial protocol. Shown are the rates of

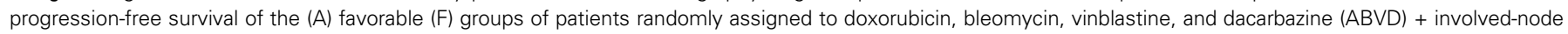

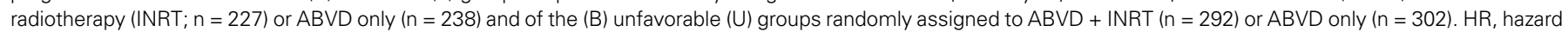
ratio, O observed; $n$, number of patients.

INRT arm and 27 patients in the BEACOPPesc + INRT arm-two thirds of the latter because of patient and/or investigator decision. Eleven percent of those who were allocated to the ABVD + INRT did not receive INRT, mainly because of progression after chemotherapy but before the start of INRT. Only $1.8 \%$ in the BEACOPPesc arm did not receive INRT.

More grade 3 and 4 hematologic toxicities were observed in the BEACOPPesc + INRT arm compared with the ABVD + INRT arm: neutropenia $(53.5 \% \quad v 30.3 \%)$, anemia $(4.9 \% \quad v$ $0.0 \%)$, and thrombocytopenia $(19.7 \% v 0.0 \%)$. Grade 3 and 4 febrile neutropenia episodes occurred in $23.9 \%$ versus $1.1 \%$ of patients, whereas infections without neutropenia were reported in $5.6 \%$ versus $1.1 \%$, respectively. Four months after the end of CMT, one patient died of pneumonitis in the BEACOPPesc arm. Altogether, 42 second malignancies were reported (Table 2).

\section{DISCUSSION}

The H10 trial was designed to evaluate an ePET response-based adaptation of treatment in patients with stage I and II HL. If ePET-positive patients could benefit from early intensification of chemotherapy, failure rates could decrease. If ePET-negative patients could be spared RT, long-term toxicity could be reduced.

After two cycles of ABVD, $18.8 \%$ of patients had a positive ePET. A significant improvement $(13.2 \%)$ of 5-year PFS was reached in the experimental BEACOPPesc + INRT arm compared with continuation with ABVD + INRT. Even a benefit in OS of $6.7 \%$, with a trend toward statistical significance, was observed. This finding is of immediate clinical relevance because on the basis of an ePET result, $<20 \%$ of patients needed intensified chemotherapy to obtain superior PFS rates after starting with ABVD. In the German Hodgkin Study Group HD 14 trial, patients with intermediate stage I and II HL were randomly assigned to either two cycles of BEACOPPesc followed by two cycles of ABVD versus four cycles of ABVD in both arms followed by involved-field RT (IFRT). ${ }^{18}$ No ePET was included in this design. This resulted in a modestly but significantly better PFS for BEACOPPesc + ABVD, but at the cost of exposing all patients from the start to BEACOPPesc. The H10 results demonstrate that $\mathrm{PET}$ response-adapted intensification is as effective as the HD14 approach, but might spare $77.6 \%$ of the $U$ patients from treatment with BEACOPPesc. The relative dose intensity of the restricted number of two cycles of BEACOPPesc was excellent and hematologic toxicities were manageable. We do not yet have results on fertility in our trial, but preliminary analysis of the HD 14 trial revealed no significant differences in female fertility potential after two cycles BEACOPPesc and two cycles of ABVD compared with four cycles of ABVD. ${ }^{19}$ The gap in time between random assignment and experimental intervention could be associated with a risk of bias in the current study; however, clinical characteristics and prognostic factors seem to be adequately balanced between both arms. In view of the significant reduction in the risk of relapse, manageable toxicity, acceptable risk of female infertility, and avoidance of second-line high-dose treatment with autologous stem cell rescue for patients who experience relapse, PET-adapted intensification in ePET-positive patients is a realistic treatment option.

ePET-negative patients had excellent outcomes, but the 5-year risk difference in PFS was $11.9 \%$ and $2.5 \%$ in favor of CMT in the $\mathrm{F}$ and $\mathrm{U}$ groups, respectively; however, for both the $\mathrm{F}$ and $\mathrm{U}$ groups, noninferiority of ABVD only could not be declared as the upper bound of the 95\% CI of the estimated HRs exceeded 


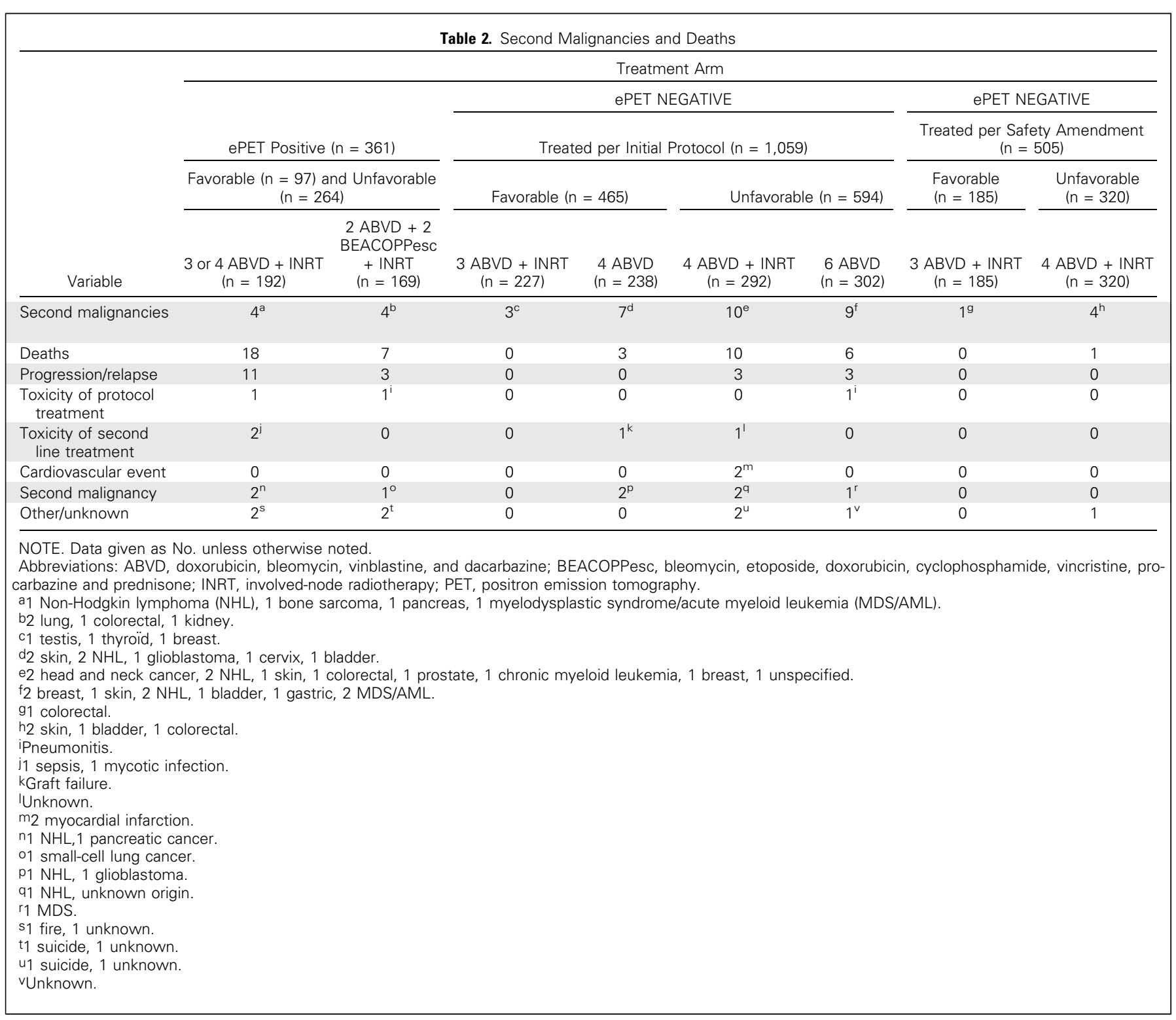

the preset noninferiority margins, which was in line with the conclusions of the interim analysis. ${ }^{11}$ Our decision to consider a $-10 \%$ difference in 5 -year PFS rates can be questioned. It reflects our attempt to balance immediate high cure rates and the risks of serious long-term toxicities. ${ }^{4,5}$ In the RAPID trial, which was designed to evaluate whether IFRT could be omitted when the PET scan after three cycles of ABVD was negative, comparable assumptions ( $-7 \%$ margin at 3 years) were adopted to balance cure rates and toxicities. ${ }^{20}$

We observed some remarkable differences between the $\mathrm{F}$ and the $\mathrm{U}$ groups. In the F group, INRT still improved disease control even in ePET-negative patients, but the overall excellent survival in both arms supports the consideration of INRT omission for selected patients in the context of individualized therapy. In the U group, 5-year PFS was only modestly impaired after omission of INRT, mainly because of several events after $>36$ months in the standard arm. This finding challenges the need for RT more explicitly in the U group. The less prominent role of INRT in patients in the $\mathrm{U}$ group compared with those in the $\mathrm{F}$ group is not evident, but perhaps the number of additional chemotherapy cycles after ePET negativity in the ABVD only arms might have played a role- two additional cycles of ABVD in the $\mathrm{F}$ group versus four in the $\mathrm{U}$ group. This assumption is supported by the analysis of relapse patterns. For patients who were treated in the experimental arms with ABVD only, relapses occurred predominantly in previously involved nodes, mainly early after end of treatment ( $\leq 2$ years). In the standard CMT arms, INRT seemed to be successful in preventing relapses in initially involved nodes as only $15.6 \%$ of patients who experienced relapse developed disease in these sites. However, in the $U$ group, which had more widespread disease than the F group, late relapses ( $>3$ years after end of treatment) occurred more frequently after CMT than after ABVD only, which possibly reflects a suboptimal amount of chemotherapy in the CMT arm. 
In the RAPID trial, including mostly favorable patients and excluding mediastinal bulk, no additional chemotherapy was administered to PET-negative patients after three cycles of $\mathrm{ABVD}$, and a comparison was made between no further treatment and IFRT. ${ }^{20}$ Here, the difference in timing of relapse between patients who received CMT and those who received chemotherapy only was not observed, which possibly reflects the influence of additional cycles of chemotherapy in the H10 trial. Long-term toxicities could not yet be incorporated in the interpretation of the outcome. This hampers the actual clinical judgment of noninferiority of a new treatment approach but is inevitable in this group of long-term survivors.

Prognostic factors, such as ESR, B symptoms, age, and bulky mediastinum defined on a chest $\mathrm{x}$-ray and used in the current study, are clearly challenged in the current era of ePET-directed therapy. The observation in the current study that 377 (72.9\%) of 517 of the bulky mediastinum (mediastinal-thoracic ratio $\geq$ 0.35 ) are PET negative after only two cycles of ABVD suggests that ePET may overcome some classic prognostic factors in localized $\mathrm{HL}$ as it overcomes the International Prognostic Score for advanced HL. ${ }^{9}$

In stage I and II HL, the outcome of patients is excellent with CMT. To improve the balance between efficacy and toxicity, ePET helps in the treatment decision for individual patients. The $\mathrm{H} 10$ shows that when ePET is positive after two cycles of ABVD, intensification with two cycles of BEACOPPesc + INRT should be considered as the best treatment option. In ePET-negative patients, the overall outcome is excellent: either after CMT or after chemotherapy only. But statistically-on the basis of our preset assumptions-noninferiority of leaving INRT out of the treatment could not be demonstrated; a negative ePET seems not to be the ideal tool to identify patients who do not need RT. In the F group, CMT results in a better immediate disease control; however, in the U group, benefit of CMT seems to be less clinically relevant, and treatment with chemotherapy only is defensible in individual patients.

\section{AUTHORS' DISCLOSURES OF POTENTIAL CONFLICTS} OF INTEREST

Disclosures provided by the authors are available with this article at jco.org.

\section{AUTHOR CONTRIBUTIONS}

Conception and design: Marc P.E. André, Théodore Girinsky, Massimo Federico, Oumédaly Reman, Catherine Fortpied, Richard van der Maazen, Christophe Fermé, Gustaaf van Imhoff, Lena Specht, Martin Hutchings, Michel Meignan, John Raemaekers

Administrative support: Valeria Fiaccadori, Monica Bellei, Tiana Raveloarivahy

Provision of study materials or patients: Marc P.E. André, Théodore Girinsky, Massimo Federico, Oumédaly Reman, Catherine Fortpied, Manuel Gotti, Olivier Casasnovas, Pauline Brice, Richard van der Maazen, Alessandro Re, Véronique Edeline, Christophe Fermé, Gustaaf van Imhoff, Francesco Merli, Réda Bouabdallah, Catherine Sebban, Lena Specht, Aspasia Stamatoullas, Richard Delarue, Annibale Versari, Martin Hutchings, Michel Meignan, John Raemaekers

Collection and assembly of data: Marc P.E. André, Massimo Federico, Oumédaly Reman, Catherine Fortpied, Manuel Gotti, Olivier Casasnovas, Pauline Brice, Richard van der Maazen, Alessandro Re, Véronique Edeline, Christophe Fermé, Gustaaf van Imhoff, Francesco Merli, Réda Bouabdallah, Catherine Sebban, Lena Specht, Aspasia Stamatoullas, Richard Delarue, Valeria Fiaccadori, Monica Bellei, Tiana Raveloarivahy, Annibale Versari, Martin Hutchings, Michel Meignan, John Raemaekers

Data analysis and interpretation: Marc P.E. André, Théodore Girinsky, Massimo Federico, Catherine Fortpied, Manuel Gotti, Olivier Casasnovas, Pauline Brice, Richard van der Maazen, Alessandro Re, Véronique Edeline, Christophe Fermé, Gustaaf van Imhoff, Francesco Merli, Réda Bouabdallah, Catherine Sebban, Lena Specht, Aspasia Stamatoullas, Richard Delarue, Valeria Fiaccadori, Tiana Raveloarivahy, Annibale Versari, Martin Hutchings, Michel Meignan, John Raemaekers

Manuscript writing: All authors

Final approval of manuscript: All authors

Accountable for all aspects of the work: All authors

\section{REFERENCES}

1. Fermé $\mathrm{C}$, Eghbali $H$, Meerwaldt $\mathrm{JH}$, et al: Chemotherapy plus involved-field radiation in earlystage Hodgkin's disease. N Engl J Med 357: 1916-1927, 2007

2. Engert $A$, Plütschow $A$, Eich $H T$, et al: Reduced treatment intensity in patients with early-stage Hodgkin's lymphoma. N Engl J Med 363:640-652, 2010

3. Brusamolino E, Anselmo AP, Klersy C, et al: The risk of acute leukemia in patients treated for Hodgkin's disease is significantly higher aft [see bined modality programs than after chemotherapy alone and is correlated with the extent of radiotherapy and type and duration of chemotherapy: A case-control study. Haematologica 83:812-823, 1998

4. Schaapveld M, Aleman BM, van Eggermond $A M$, et al: Second cancer risk up to 40 years after treatment for Hodgkin's lymphoma. N Engl J Med 373:2499-2511, 2015

5. Hancock SL, Tucker MA, Hoppe RT: Factors affecting late mortality from heart disease after treatment of Hodgkin's disease. JAMA 270:1949-1955, 1993

6. De Bruin ML, Dorresteijn LD, van't Veer MB, et al: Increased risk of stroke and transient ischemic attack in 5-year survivors of Hodgkin lymphoma. J Natl Cancer Inst 101:928-937, 2009

7. Meyer RM, Gospodarowicz MK, Connors JM, et al: ABVD alone versus radiation-based therapy in limited-stage Hodgkin's lymphoma. N Engl J Med 366:399-408, 2012

8. Rigacci $L$, Puccini $B$, Zinzani $P L$, et al: The prognostic value of positron emission tomography performed after two courses (INTERIM-PET) of standard therapy on treatment outcome in early stage Hodgkin lymphoma: A multicentric study by the Fondazione Italiana Linfomi (FIL). Am J Hematol 90:499-503, 2015

9. Gallamini $A$, Hutchings $M$, Rigacci $L$, et al: Early interim 2-[18 F]fluoro-2-deoxy-D-glucose positron emission tomography is prognostically superior to international prognostic score in advanced-stage Hodgkin's lymphoma: A report from a joint ItalianDanish study. J Clin Oncol 25:3746-3752, 2007

10. Hutchings $M$, Loft $A$, Hansen $M$, et al: FDGPET after two cycles of chemotherapy predicts treatment failure and progression-free survival in Hodgkin lymphoma. Blood 107:52-59, 2006

11. Raemaekers JM, André MP, Federico M, et al: Omitting radiotherapy in early positron emission tomography-negative stage I/II Hodgkin lymphoma is associated with an increased risk of early relapse: Clinical results of the preplanned interim analysis of the randomized EORTC/LYSA/FIL H10 trial. J Clin Oncol 32:1188-1194, 2014

12. Cheson BD, Horning SJ, Coiffier B, et al: Report of an international workshop to standardize response criteria for non-Hodgkin's lymphomas. J Clin Oncol 17:1244, 1999

13. Diehl V, Franklin J, Pfreundschuh $M$, et al: Standard and increased-dose BEACOPP chemotherapy compared with COPP-ABVD for advanced Hodgkin's disease. N Engl J Med 348:2386-2395, 2003 [Erratum: N Engl J Med 353:744, 2005]

14. Girinsky $T$, Specht $L$, Ghalibafian $M$, et al: The conundrum of Hodgkin lymphoma nodes: To be or not to be included in the involved node radiation fields. The EORTC-GELA lymphoma group guidelines. Radiother Oncol 88:202-210, 2008

15. Girinsky T, van der Maazen R, Specht L, et al: Involved-node radiotherapy (INRT) in patients with 
early Hodgkin lymphoma: Concepts and guidelines. Radiother Oncol 79:270-277, 2006

16. Juweid ME, Stroobants $S$, Hoekstra OS, et al: Use of positron emission tomography for response assessment of lymphoma: Consensus of the Imaging Subcommittee of International Harmonization Project in Lymphoma. J Clin Oncol 25:571-578, 2007

17. Meignan $M$, Itti $E$, Bardet $S$, et al: Development and application of a real-time on-line blinded independent central review of interim PET scans to determine treatment allocation in lymphoma trials. J Clin Oncol 27:2739-2741, 2009

18. von Tresckow $B$, Plütschow $A$, Fuchs $M$, et al: Dose-intensification in early unfavorable Hodgkin's lymphoma: Final analysis of the German Hodgkin Study Group HD14 trial. J Clin Oncol 30:907-913, 2012
19. Behringer $K$, Thielen I, Mueller H, et al: Fertility and gonadal function in female survivors after treatment of early unfavorable Hodgkin lymphoma (HL) within the German Hodgkin Study Group HD14 trial. Ann Oncol 23:1818-1825, 2012

20. Radford J, Illidge T, Counsell N, et al: Results of a trial of PET-directed therapy for early-stage Hodgkin's lymphoma. N Engl J Med 372:1598-1607, 2015

\section{Affiliations}

Marc P.E. André, Université Catholique de Louvain, Yvoir; Catherine Fortpied, Valeria Fiaccadori, and Tiana Raveloarivahy, European Organisation for Research and Treatment of Cancer, Brussels, Belgium; Théodore Girinsky and Christophe Fermé, Institut Gustave Roussy, Villejuif; Oumédaly Reman, Institut d'Hématologie de Basse Normandie, Centre Hospitalier Universitaire, Caen; Pauline Brice, Assistance Publique des Hopitaux de Paris Hôpital Saint-Louis; Richard Delarue, Assistance Publique des Hopitaux de Paris Hôpital Universitaire Necker-Enfants Maladies, Paris; Olivier Casasnovas, Centre Hospitalier Universitaire le Bocage and Institut National de la Santé et de la Recherche Médicale, Dijon; Véronique Edeline, Hôpital René Hugenin-Institut Curie, Saint Cloud; Réda Bouabdallah, Institut Paoli Calmette, Marseille; Catherine Sebban, Hematology Centre Léon Bérard, Lyon; Aspasia Stamatoullas, Centre Henri Becquerel, Rouen; Michel Meignan, Henri Mondor University Hospitals, Créteil, France; Massimo Federico and Monica Bellei, University of Modena and Reggio Emilia, Modena; Manuel Gotti, Fondazione Istituto di Ricovero e Cura a Carattere Scientifico Policlinico San Matteo, Pavia; Alessandro Re, Spedali Civili Hospital, Brescia; Francesco Merli and Annibale Versari, Arcispedale Santa Maria Nuova Istituto di Ricovero e Cura a Carattere Scientifico, Reggio Emilia, Italy; Richard van der Maazen and John Raemaekers, Radboud University Medical Center, Nijmegen; Gustaaf van Imhoff, University of Groningen, University Medical Centre Groningen, Groningen, the Netherlands; and Lena Specht and Martin Hutchings, Rigshospitalet, Copenhagen University Hospital, Copenhagen, Denmark

\section{Support}

Supported by European Organisation for Research and Treatment of Cancer (Belgium), LYmphoma Study Association (France), Fondazione Italiana Limfomi (Italy), Fondation Belge contre le Cancer (Belgium), Dutch Cancer Society (the Netherlands), Institut National du Cancer (France), Assistance Publique des Hopitaux de Paris (France), Societe Française de Medecine Nucleaire et Imagerie Moleculaire (France), Associazone Angela Serra (Italy), van Vlissingen Lymfoom Fonds (the Netherlands), and Chugai Pharmaceutical (Japan).

\section{Prior Presentation}

Presented at the 51st Annual Meeting of the American Society of Hematology, New Orleans, LA, December 5-8, 2009; the Eighth International Symposium on Hodgkin Lymphoma, Cologne, Germany, October 23-26, 2010; the 54th Annual Meeting of the American Society of Hematology, Atlanta, GA, December 8-11, 2012; the International Conference on Malignant Lymphoma, Lugano, Switzerland, June 17-20, 2015; and the 10th International Symposium on Hodgkin Lymphoma, Cologne, October 22-25, 2016. 


\section{AUTHORS' DISCLOSURES OF POTENTIAL CONFLICTS OF INTEREST}

Early Positron Emission Tomography Response-Adapted Treatment in Stage I and II Hodgkin Lymphoma: Final Results of the Randomized EORTC/LYSA/FIL H10 Trial

The following represents disclosure information provided by authors of this manuscript. All relationships are considered compensated. Relationships are self-held unless noted. I = Immediate Family Member, Inst = My Institution. Relationships may not relate to the subject matter of this manuscript. For more information about ASCO's conflict of interest policy, please refer to www.asco.org/rwc or ascopubs.org/jco/site/ifc.

Marc P.E. André

No relationship to disclose

Théodore Girinsky

No relationship to disclose

Massimo Federico

No relationship to disclose

Oumédaly Reman

No relationship to disclose

Catherine Fortpied

No relationship to disclose

\section{Manuel Gotti}

No relationship to disclose

\section{Olivier Casasnovas}

Honoraria: Genentech, Takeda, Gilead Sciences, Sanofi

Consulting or Advisory Role: Genentech, Takeda, Gilead Sciences

Research Funding: Genentech (Inst)

Travel, Accommodations, Expenses: Genentech, Takeda, Gilead Sciences

Pauline Brice

Research Funding: Merck Sharp \& Dohme Oncology (Inst), Takeda (Inst) Travel, Accommodations, Expenses: Takeda

Richard van der Maazen

No relationship to disclose

Alessandro Re

No relationship to disclose

Véronique Edeline

No relationship to disclose

Christophe Fermé

No relationship to disclose

Gustaaf van Imhoff

No relationship to disclose

Francesco Merli

No relationship to disclose
Réda Bouabdallah

No relationship to disclose

\section{Catherine Sebban}

No relationship to disclose

Lena Specht

Consulting or Advisory Role: Takeda

Research Funding: Varian Medical Systems

Travel, Accommodations, Expenses: Takeda

Aspasia Stamatoullas

No relationship to disclose

Richard Delarue

Honoraria: Servier, Gilead Sciences, Roche, Celgene, Takeda

Consulting or Advisory Role: Gilead Sciences, Roche

Speakers' Bureau: Karyopharm Therapeutics

Travel, Accommodations, Expenses: Roche, Takeda, Celgene

Valeria Fiaccadori

No relationship to disclose

Monica Bellei

No relationship to disclose

Tiana Raveloarivahy

No relationship to disclose

Annibale Versari

No relationship to disclose

Martin Hutchings

Consulting or Advisory Role: Takeda, Genentech, Celgene, Bayer

Research Funding: Takeda (Inst), Janssen-Cilag (Inst), Genentech (Inst), Celgene (Inst)

Travel, Accommodations, Expenses: Takeda, Bristol-Myers Squibb, Janssen-Cilag

\section{Michel Meignan}

No relationship to disclose

John Raemaekers

No relationship to disclose 


\section{Acknowledgment}

We thank the patients and their families for participation to this study. We also thank Liliana Baila, Sabine de Bedout, Jocelyne Flament, Matthias Karrasch, Emad Shash and Safaa Ramadan from European Organisation for Research and Treatment of Cancer Headquarters and Anissa Zarour from Assistance Publique-Hôpitaux de Paris for their contribution to the present study.

\section{Participating Centers}

Belgium: F. Offner, Universitair Ziekenhuis (UZ) Gent; A. Van Hoof, AZ St. Jan; R. De Bock, K. Beel, ZNA Middelheim; A. Efira, Hôpital Universitaire Brugmann; P. Zachee, Ziekenhuis Netwerk Antwerpen Stuivenberg; W.A. Schroyens, UZ Antwerpen; A. Ferrant, Cliniques Universitaires Saint-Luc; A. Bosly, Centre Hospitalier Universitaire (CHU) Dinant Godinne, UCL Namur; A. Kentos, Hôpitaux Universitaires Bordet-Erasme-Hôpital Universitaire Erasme; A. Triffet, CHU de Charleroi-Hôpital André Vésale; S. Vansteenweghen, Centre Hospitalier Régional de la Citadelle; G.E.G. Verhoef, Y. Lievens, UZ Leuven, Campus Gasthuisberg; D. Bron, Hôpitaux Universitaires Bordet-Erasme-Institut Jules Bordet; B. De Prijck, CHU Sart-Tilman; N. Straetmans, Hôpital de Jolimont; H. Demuynck, AZ Delta-Heilige Hartziekenhuis Roeselare, Menen; V. Mathieux, Clinique et Maternité Sainte Elisabeth; M. André, Grand Hôpital de Charleroi, Site Notre-Dame; T. Connerotte, Clinique Saint-Pierre; D. Boulet, Centre Hospitalier Régional-Clinique Saint-Joseph; P. Mineur, Grand Hôpital de Charleroi-Hôpitaux Saint-Joseph-Sainte-Thérèse-IMTR; M. Maerevoet, Clinique Notre-Damede Grâce; P. Pierre, Cliniques du Sud Luxembourg-Clinique Saint-Joseph. Croatia: I. Aurer, University Hospital Rebro. Denmark: L. Specht, Rigshospitalet. France: M. Azagury, Centre Hospitalier $(\mathrm{CH})$ de Saint Germain; C. Haouin, Assistance Publique-Hôpitaux de Paris (APHP)-CHU Henri Mondor; M. Aoudjhane, APHP-Hôpital Saint Antoine; C. Fruchart, Centre Régional François Baclesse; A. Stamatoullas-Bastard, Centre Henri Becquerel; F. Boue, APHP-Hôpital Antoine Beclere; C. Kelaidi, APHP-Hôpital Avicenne; A. Thyss, Centre Antoine Lacassagne, G. Laurent, CHU de Toulouse-Hôpital de Purpan; S. Cailleres, CH d'Aix-en-Provence; C. Ferme, Institut Gustave Roussy, C. Sebban, Centre Léon Bérard; H. Eghbali, Institut Bergonie, J. Gabarre, APHP-La Pitié Salpétrière; A. Devidas, CH Sud Francilien-Gilles De Corbeil; S. Natarajan-Ame, Hôpitaux Universitaires de Strasbourg-Hautepierre; A. Delmer, APHP-Hôtel-Dieu de Paris; F. Dreyfus, APHP-Hôpital Cochin; S. Glaisner, Institut Curie-Hôpital René Huguenin; F. Morschhauser, Centre Hospitalier Regional Universitaire (CHRU) Lille; D. Decaudin, Institut Curie; D. Jaubert, Hôpital Bellevue; R. Delarue, APHP-Hôpital Necker; N. Ali Amar, CHU de Troyes; O. Casasnovas, CHU de Dijon; R. Bouabdallah, Institut J. Paoli \& I. Calmettes; O. Tournilhac, Nouvel Hôpital Estaing; G. Sebahoun, Assistance Publique-Hôpitaux de Marseille-Hôpital Nord; B. Christian, Hôpital Notre-Dame de Bon Secours; P. Helias, CHRU de Besançon- Hôpital Jean Minjoz; M. Fabbro, Institut Régional du Cancer Montpellier; P. Brice, APHP-Hôpital SaintLouis; K. Bouabdallah, CHU de Bordeaux-Group Hospitalier Sud-Hôpital Haut-Lévêque; V. Morel, CH Victor Dupouy; B. Coiffier, CHU de Lyon-CH Lyon Sud; S. Bologna, CHU de Nancy-Hôpitaux De Brabois; D. Bordessoule, CHU de Limoges-Hôpital Dupuytren; B. Corront, CH d'Annecy; W. Abarah, CH de Meaux; F. Bauduer, CH de la Côte Basque; C. Bresson, APHP-Hôpital de Bicetre; B. Salles, Université Paul Sabatier-Bâtiment Centre Interuniversitaire de Recherche et d'Ingénierie des Matériaux-Centre National de la Recherche Scientifique; E. Fleck, Hôpital Saint-Louis; S. Castaigne, CH de Versailles-Hôpital André Mignot; M. Blanc, CH de Chambery; H. Gonzales, CH René Dubos; H. Orfeuvre, CH de Bourg-en-Bresse; J.M. Karsenti, CHU de Nice-Hôpital de l'Archet; O. Reman, CHU de Caen-Hôpital Clémenceau; J.P. Marolleau, CHU d'Amiens, Hôpital Sud; Souleau, Hôpital d'Instruction des Armées Percy; L. Al JassemAbd El Kader, Hôpital Louis Pasteur (ex Fontenoy); D. Assouline, Clinique du Mail; C. Kulekci, CH Marc Jacquet; O. Fitoussi, Polyclinique de Bordeaux-Nord Aquitaine; J.C. Eisenmann, CH de Mulhouse, Hôpital Emile Muller-Moenchsberg; J.M. Pignon, CH de Dunkerque; R. Garidi, Hôpital Saint Quentin; X. Levaltier, Clinique du Parc; I. Plantier, CH de Roubaix-Hôpital V Provo; L. Detourmignies, CH de Roubaix-Hôpital V Provo. Italy: J. Leone, Policlinico Universitario A. Gemelli-Universita del Sacro Cuore; A. Zaccaria, Ospedale Santa Maria dell Croci; L. Gargantini, Ospedale Niguarda Ca Granda; G. Pinotti, Ospedale Di Circolo E Fondazione Macchi; U. Vitolo, P. Gavarotti, Azienda Ospedaliera Citta della Salute et della Scienza di Torino-Ospedale Molinette; F. Merli, Arcispedale di S. Maria Nuova; M. Martelli, A. Pulsoni, Universita di Roma "La Sapienza"-Clinica Ematologica de l’Universita di Roma "La Sapienza"; P.L Zinzani, Universita di Bologna; M. Petrini, Azienda Ospedaliera Universitaria Pisana; F. Zaja, Azienda Ospedaliera-Universitaria "Santa Maria della Misericordia” di Udine; M. Spina, Centro di Riferimento Onconlogico; J.M. Ferreri Andres, Ospedale San Raffaele; C. Petti, Istituto Regina Elena/Istituo Fisioterapici Ospitalieri; E. Pogliani, Ospedale San Gerardo; S. Barra, Istituto Nazionale per la Ricerca sul Cancro; A. Rovati, Ospedale Maggiore di Lodi, A.M Carella, Istituto di Ricovero e Cura a Carattere Scientifico Azienda Ospedaliera Universitaria San Martino "IST" Istituto Nazionale per la Ricerca sul Cancro; F. Nobile, Azienda Ospedaliera Bianchi-Melacrino-Morelli; E. Abruzzese, Ospedale San Eugenio; V. Rissoli, Azienda Ospedaliera di Parma; S. Molica, Ospedale Regionale A. Pugliese; R. Cantaffa, Ospedale Regionale A. Pugliese; E. Brusamolino, Policlinico San Matteo; E. Angelucci, Ospedale Oncologico A. Busino; M. Lombardo, F. Angrilli, Ospedale Civile Pescara; M. Federico, Azienda Ospedaliero-Universitaria Policlinico di Modena; M. Longinotti, Istituto di Ematologia-Universita di Sassari; P.P. Fattori, Ospedale Infermi di Rimini; A. Gallamini, Ospedale Santa Croce; M. Girotto, Ospedale Civile Ivrea; N. Cascavilla, Ospedale Sollievo Della Sofferenza; V. Liso, Universita Degli Studi di Bari-Policlinico; G. Rossi, Universita di Brescia-Azienda Ospedaliera Spedali Civili di Brescia; L. Baldini, Istituto di Igiene, Universita di Milano; A. Levis, Ospedale Civile; C. Rosanelli, Ospedale Generale Regionale; P. Guglielmo, Ospedale Garibaldi-Universita di Catania; G. Quarta, Ospedale Regionale A. di Summa; A. Santoro, Istituto Clinico 
Humanitas; M. Aglietta, Fondazione del Piemonte per l'Oncologia-Istitute for Cancer Research and Treatment; A. Fabbri, Universita Delli Studi di Siena-Policlinico "le Scotte"; M. Musso, La Maddalena S.P.A.; M. Brugiatelli, Azienda Ospedaliera Papardo; A.M. Darco, Ospedale Civile Umberto I; A.M. Liberati, Azienda Ospedaliera S. Maria; M.C. Cox, Universita di Roma "La Sapienza”-Universita Degli Studi di Roma “La Sapienza”-Ospedale Sant'Andrea; G. Gaidano, Amedeo Avogadro University of Eastern Piedmont-Ospedale Maggiore della Carita; F. Morabito, A.O. di Cosenza; L. Flenghi, Santa Maria della Misericordia Hospital; D. Amadori, Istituto Scientifico Romagnolo per lo Studio et la Cura dei Tumori; G. Giglio, Fondazione di Ricerca et Cura Giovanni Paolo II-Universita Cattolica del Sacro Cuore; G. Partesotti, Ospedale Civile Sassuolo; D. Vallisa, Ospedale Civile Piacenza. Netherlands: G.J. Goverde, Amphia Ziekenhuis (Molengracht); M. Silbermann, Tergooiziekenhuizen (Blaricum); E.F.M. Posthuma, Reinier De Graaf Gasthuis; H.A.M. Sinnige, Jeroen Bosch Ziekenhuis; M.L.M. Lybeert, Catharina Ziekenhuis; F. Heyning, Medisch Centrum Haaglanden-Antoniushove; F. Peters, Maaslandziekenhuis; J.W. Baars, The Netherlands Cancer Institute-Antoni Van Leeuwenhoeziekenhuis; O.S. Leeksma, Onze Lieve Vrouw Gasthuis; F. Ong, Medisch Spectrum Twente; G.K.S. Jie, Atrium Medisch Centrum; H. Schouten, Academisch Ziekenhuis Maastricht; E.C. Dompeling, Isala Klinieken (Sophia); M.R. Schipperus, HagaZiekenhuis (Leyweg); P.J. Lugtenburg, Erasmus MC Hospital; M.O. Den Boer, Laurentius Ziekenhuis Roermond; P.J. Lugtenburg, Erasmus MC Cancer Institute (Daniel den Hoed); J. Raemaekers, Radboud University Medical Center Nijmegen; A.D.G. Krol, Leiden University Medical Centre; G.W. Van Imhoff, University Medical Center Groningen; M.J. Kersten, Academisch Medisch Centrum-Universiteit van Amsterdam; R. Van Der Griend, E.V. Planken, Diaconessenhuis; R. Fijnheer, Meander Medisch Centrum de Lichtenberg; D.H. Biesma, H.R. Koene, St. Antonius Ziekenhuis; W. Smit, Radiotherapeutisch Instituut Friesland; P. Joosten, M. Hoogendoorn, Medisch Centrum Leeuwarden-Zuid. Slovakia: A. Vranovsky, National Cancer Institute. Switzerland: G. Berthod, CHU Vaudois-Lausanne.

\section{Appendix}

A

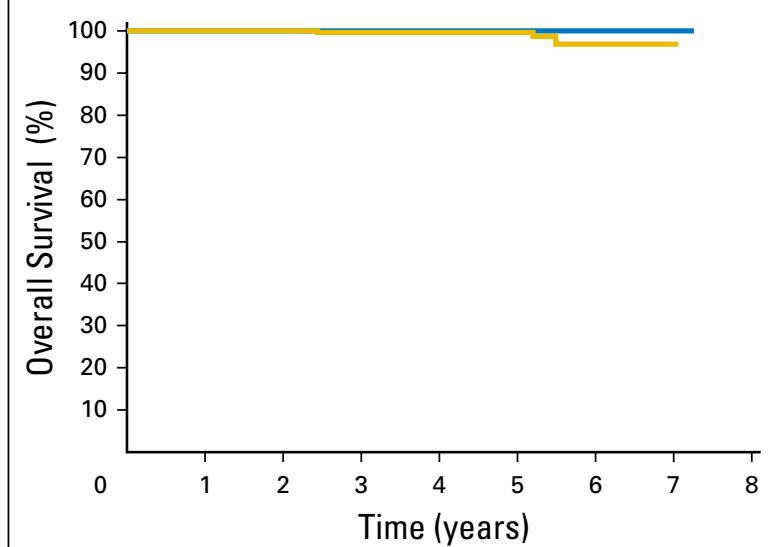

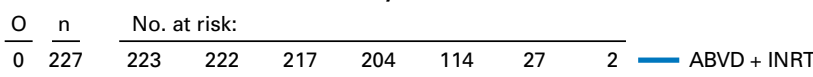

B

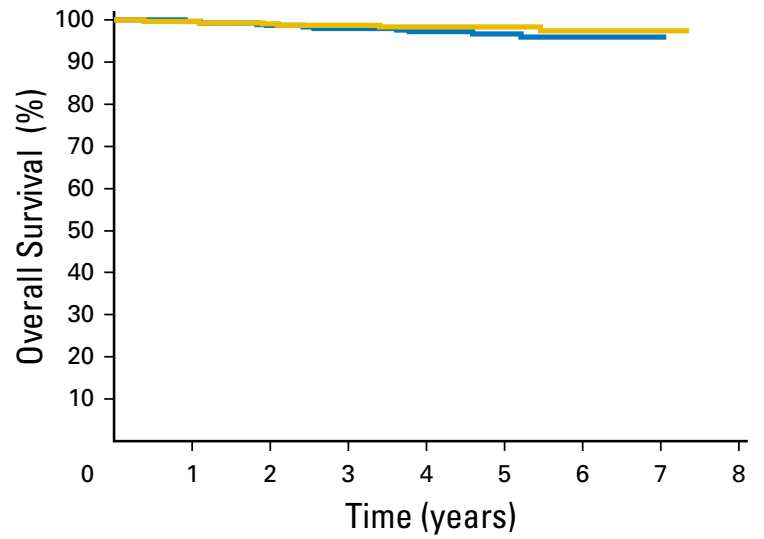

\begin{tabular}{|c|c|c|c|c|c|c|c|c|c|}
\hline 0 & $\mathrm{n}$ & No. & risk: & & & & & & \\
\hline 10 & 292 & 289 & 283 & 274 & 256 & 154 & 38 & 3 & ABVD + INRT \\
\hline 6 & 302 & 299 & 290 & 285 & 264 & 156 & 43 & 4 & ABVD only \\
\hline
\end{tabular}

Fig A1. Overall survival of the 1,059 early positron emission tomography-negative patients (treated per initial protocol). Shown are the rates of (A) overall survival in the

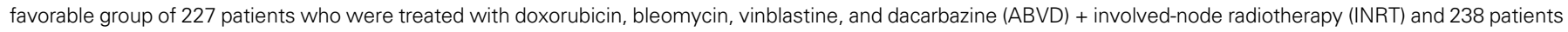

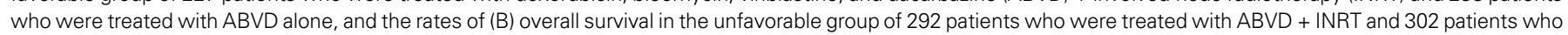
were treated with ABVD alone, O observed; $n$, number of patients. 


\begin{tabular}{|c|c|c|c|c|c|c|}
\hline \multirow[b]{3}{*}{ End Point and Population } & \multirow{2}{*}{\multicolumn{2}{|c|}{$\begin{array}{c}\text { PET Positive } \\
\text { Favorable and Unfavorable }\end{array}$}} & \multicolumn{4}{|c|}{ PET Negative } \\
\hline & & & \multicolumn{2}{|c|}{ Favorable } & \multicolumn{2}{|c|}{ Unfavorable } \\
\hline & 3 or 4 ABVD + INRT & $\begin{array}{c}2 \text { ABVD + } 2 \\
\text { BEACOPPesc + INRT }\end{array}$ & 3 ABVD + INRT & 4 ABVD & 4 ABVD + INRT & 6 ABVD \\
\hline \multicolumn{7}{|l|}{ PFS } \\
\hline \multicolumn{7}{|l|}{ Per-protocol } \\
\hline No. & 172 & 159 & 221 & 232 & 268 & 289 \\
\hline $\begin{array}{c}\% \text { at } 5 \text { years } \\
(95 \% \mathrm{Cl})\end{array}$ & 75.9 (68.4 to 81.9$)$ & 91.9 (86 to 95.4$)$ & 98.9 (95.7 to 99.7$)$ & 88.6 (83.6 to 92.1$)$ & 91.7 (87.3 to 94.6) & 89.8 (85.7 to 92.8 ) \\
\hline $\mathrm{HR}^{*}(95 \% \mathrm{Cl})$ & $0.33(0$ & 8 to 0.62 ) & $13.7(3.2$ & to 57.55$)$ & $1.32(0.7$ & to 2.31 ) \\
\hline$P$ & $<$ & & & $6+$ & & $8+$ \\
\hline \multicolumn{7}{|l|}{ Best scenario } \\
\hline No. & & & 229 & 239 & 299 & 303 \\
\hline $\begin{array}{c}\% \text { at } 5 \text { years } \\
(95 \% \mathrm{Cl})\end{array}$ & & & 99.0 (95.9 to 99.7 ) & 86.8 (81.7 to 90.5$)$ & 91.5 (87.5 to 94.3 ) & 89.6 (85.6 to 92.6 ) \\
\hline HR $(95 \% \mathrm{Cl})$ & & & 16.4 & to 68.45$)$ & $1.34(0.7$ & to 2.28 ) \\
\hline$P$ & & & & $38+$ & .95 & \\
\hline \multicolumn{7}{|l|}{ Worst scenario } \\
\hline & 203 & 171 & & & & \\
\hline $\begin{array}{l}\% \text { at } 5 \text { years } \\
(95 \% \mathrm{Cl})\end{array}$ & 76.9 (70.1 to 82.3 ) & 90.1 (84.2 to 93.9) & & & & \\
\hline $\mathrm{HR}(95 \% \mathrm{Cl})$ & $0.43(0$ & 4 to 0.75$)$ & & & & \\
\hline$P$ & & & & & & \\
\hline \multicolumn{7}{|l|}{ OS } \\
\hline \multicolumn{7}{|l|}{ Per-protocol } \\
\hline No. & 172 & 159 & 221 & 232 & 292 & 302 \\
\hline $\begin{array}{c}\% \text { at } 5 \text { years } \\
(95 \% \mathrm{Cl})\end{array}$ & 89.3 (82.9 to 93.4$)$ & 95.7 (90.5 to 98.1$)$ & 100.0 & 99.6 (96.9 to 99.9 ) & 96.7 (92.9 to 97.8) & 98.3 (96.0 to 99.3 ) \\
\hline $\mathrm{HR}^{*}(95 \% \mathrm{Cl})$ & $0.48(0.2$ & to 1.16$)$ & & /A & 0.4610 .1 & to 1.35$)$ \\
\hline$P$ & & 97 & & /A & $\mathrm{N}$ & \\
\hline \multicolumn{7}{|l|}{ Best scenario } \\
\hline No. & & & 229 & 239 & 299 & 303 \\
\hline $\begin{array}{c}\% \text { at } 5 \text { years } \\
(95 \% \mathrm{Cl})\end{array}$ & & & 100.0 & 99.6 (97.0 to 99.9 ) & 96.0 (92.9 to 97.8 ) & 98.3 (96.0 to 99.3 ) \\
\hline $\mathrm{HR}^{*}(95 \% \mathrm{Cl})$ & & & & /A & $0.48(0.1 \varepsilon$ & to 1.29 ) \\
\hline$P$ & & & & /A & $\mathrm{N}$ & \\
\hline \multicolumn{7}{|l|}{ Worst scenario } \\
\hline & 203 & 171 & & & & \\
\hline $\begin{array}{c}\% \text { at } 5 \text { years } \\
(95 \% \mathrm{Cl})\end{array}$ & 88.7 (82.8 to 92.6) & 96.0 (91.2 to 98.2 ) & & & & \\
\hline$H R^{*}(95 \% \mathrm{Cl})$ & $0.41(0.1$ & to 0.97 ) & & & & \\
\hline$P$ & & 35 & & & & \\
\hline $\begin{array}{l}\text { NOTE. Per-protocol popula } \\
\text { wrong risk group (favorable } \\
\text { excluding patients who hav } \\
\text { those two cycles but for w } \\
\text { Abbreviations: BEACOPPe } \\
\text { radiotherapy; N/A, not appli } \\
{ }^{*} \text { Experimental } v \text { standard } \\
+P \text { value corresponding to }\end{array}$ & $\begin{array}{l}\text { on: All study patients w } \\
\text { unfavorable; positron e } \\
\text { not completed the firs } \\
\text { om no PET scan was } \\
\text { c, bleomycin, etoposid } \\
\text { able; OS, overall surviv } \\
\text { rm. } \\
\text { oninferiority test. }\end{array}$ & $\begin{array}{l}\text { o were eligible for the tri } \\
\text { hission tomography [PE } \\
\text { two cycles of doxorubic } \\
\text { erformed are respective } \\
\text { doxorubicin, cyclophos } \\
\text { l; PFS, progression-free }\end{array}$ & $\begin{array}{l}\text { In addition, we exclu } \\
\text { negative or positive } \\
\text {, bleomycin, vinblast } \\
\text { classified as PET ne } \\
\text { namide, vincristine, p } \\
\text { urvival. }\end{array}$ & $\begin{array}{l}\text { ded from this populati } \\
\text { Best and worst scer } \\
\text { ne, and dacarbazine ( } \\
\text { gative (best scenario) } \\
\text { ocarbazine and predn }\end{array}$ & $\begin{array}{l}\text { n patients who were } \\
\text { ario populations: Inte } \\
\text { BVD) cycles; patients } \\
\text { and PET positive (wor } \\
\text { sone; HR, hazard ratio }\end{array}$ & $\begin{array}{l}\text { ater found to be in the } \\
\text { tt-to-treat population, } \\
\text { who have completed } \\
\text { st scenario). } \\
\text { INRT, involved-node }\end{array}$ \\
\hline
\end{tabular}

